\title{
Using the Mouse to Model Human Diseases: Cognitive Impairment in Systemic Lupus Erythematosus
}

\author{
Lara El Khoury, Aida Zarfeshani ${ }^{\circledR}$, and Betty Diamond
}

\begin{abstract}
In this 2020 Dunlop-Dottridge Lecture, the authors discuss cognitive impairment (CI), one of the most prevalent neuropsychiatric syndromes in systemic lupus erythematosus (SLE). Patients often report CI as the most bothersome disease-related manifestation, with a great effect on their quality of life. Nevertheless, studies focusing on CI remain scarce and no effective targeted therapy has been identified. We herein present murine models of CI in SLE with insights into the pathogenesis of this condition as well as the role of the renin angiotensin system in microglial activation. We will discuss the role of neuroimaging as a useful objective assessment tool, describing our experience in previous and ongoing clinical trials of CI in patients with SLE. (J Rheumatol 2020;47:1145-9; doi:10.3899/ jrheum.200410)
\end{abstract}

Key Indexing Terms:

MURINE MODELS COGNITIVE IMPAIRMENT SYSTEMIC LUPUS ERYTHEMATOSUS

Cognitive impairment (CI) is one of the 19 syndromes that constitute neuropsychiatric systemic lupus erythematosus $(\mathrm{NPSLE})^{1,2}$. The term refers to deficits in any cognitive domain such as learning and executive skills, psychomotor function, attention and memory ${ }^{1}$. The prevalence of CI in SLE is highly variable, ranging from 6.6 to $80 \%{ }^{2,3}$. This wide range can be attributed to the variability in assessments and measures between different reports, the lack of a standardized definition, and most of all the difficulty attributing CI uniquely to SLE.

CI tends to develop insidiously over the course of the disease, independent from other SLE clinical manifestations. It has been shown that patients with CI tend to have higher unemployment rates and decreased quality of life (QOL) ${ }^{4,5}$. During a patient-focused drug development meeting in 2017, individuals with SLE selected forgetfulness and lack of concentration as causing substantial harm to their lives. Most survey respondents referred to these symptoms as "brain fog"6.

There are multiple barriers to studying CI in SLE including the lack of a standardized objective assessment and screening tools. Confounding factors that make it difficult to

From the Institute of Molecular Medicine, The Feinstein Institutes for Medical Research, Northwell Health, Manhasset, New York, USA.

L. El Khoury, MD, Institute of Molecular Medicine, The Feinstein Institutes for Medical Research, Northwell Health; A. Zarfeshani, PhD, Institute of Molecular Medicine, The Feinstein Institutes for Medical Research, Northwell Health; B. Diamond, MD, Institute of Molecular Medicine, The Feinstein Institutes for Medical Research, Northwell

Health. Dr. El Khoury and Dr. Zarfeshani are co-first authors who contributed equally to this report.

Address correspondence to Dr. B. Diamond, Institute for Molecular

Medicine, The Feinstein Institutes for Medical Research,

350 Community Drive, Manhasset, New York 11030, USA.

E-mail: bdiamond@northwell.edu attribute CI exclusively to SLE, such as inclusion of patients with focal NPSLE manifestations for studies. Reconciling these obstacles with the wide prevalence of CI and its major effect on the QOL of patients with SLE is a major unmet need.

\section{Murine Models of CI in NPSLE}

Mouse models of disease can offer insight into pathogenesis. Most of the SLE mouse models develop antinuclear antibodies (ANA) and immune complex glomerulonephritis. Some murine models of SLE have been assessed for cognitive dysfunction. They provide insights into underlying mechanisms that might be comparable to those in patients with SLE.

\section{Spontaneous Mouse Models}

The most widely studied spontaneous models of SLE are the New Zealand Black crossed with the New Zealand White mouse (NZB/W), BXSB/Yaa, and MRL/lpr strains.

Female NZB/W mice make ANA and anti-dsDNA autoantibodies and develop glomerulonephritis ${ }^{7}$. The study of this strain is difficult because the time to onset of disease is long and the 2 strains need to be bred together to have offspring for study. Similar to humans with SLE, they exhibit learning impairments and mood-disorder behaviors ${ }^{8}$, and the incidence and severity of SLE is greater in females ${ }^{9}$.

The BXSB/Yaa mouse model differs from others because disease is presented only in male mice. The disease depends on the Y-autoimmune accelerator (Yaa) locus, which is translocated from a region of the $\mathrm{X}$ chromosome to the $\mathrm{Y}$ chromosome ${ }^{10,11}$. This region contains 16 genes including Toll-like receptor (TLR)7. TLR7 overexpression leads to activation of the type 1 interferon (IFN) pathway, a critical pathogenic

Personal non-commercial use only. The Journal of Rheumatology Copyright $\subset$ 2020. All rights reserved. 
pathway in SLE ${ }^{12}$. The affected mice develop an SLE-like disease with ANA, glomerulonephritis, and impairment in spatial memory ${ }^{8}$.

The MRL/lpr mouse develops an SLE-like disease at a younger age than NZB/W mice. The $L P R$ gene leads to loss of Fas function ${ }^{13,14}$; however, Fas-deficient humans do not develop an SLE-like phenotype. Similarly, breeding of $L P R$ into $\mathrm{B} 6, \mathrm{Balb} / \mathrm{c}, \mathrm{AKR}$, and $\mathrm{C} 3 \mathrm{H}$ mice leads to very mild autoimmune disease ${ }^{15,16}$. The MRL+ mice develop SLE phenotype at 18 months of age; thus they are useful for studying accelerants of the disease ${ }^{17,18}$. Manifestations of SLE include ANA, glomerulonephritis, and cognitive dysfunction (anxiety, depression, anhedonia, decreased locomotion, and impaired spatial learning $)^{8}$.

\section{Induced Mouse Models}

Human SLE is believed to be triggered by environmental factors in genetically susceptible individuals; thus, the study of induced models of SLE is also useful.

Pristane is an isoprenoid alkane abundant in mineral oil. Intraperitoneal injection of pristane to Balb/c mice develops an SLE-like disease with ANA and immune complex-mediated glomerulonephritis with high levels of IFN. It also results in downregulation of the GluN2A subunit of the NMDA receptor in hippocampal neurons and to disrupted learning and memory deficits ${ }^{19}$. Abnormal levels of IFN- $\alpha$ have been observed in the sera and cerebrospinal fluid (CSF) of patients with mental disorders ${ }^{20,21}$. Intravenous injection of IFN- $\alpha$ leads to anxiety and depression-like behaviors as well as CI in female NZB/W/ mice.

We have developed an induced model of SLE that requires immunization of non-spontaneously autoimmune mice with a peptide mimetope of DNA (DWEYS) ${ }^{22}$. Immunization with a multimeric form of this sequence results in production of anti-DNA antibodies, immunoglobulin deposition in the kidneys, and cognitive dysfunction in mice in which an antibody can penetrate brain parenchyma. We will describe this model in detail. The anti-DNA antibodies in this model, termed DNRAb, cross-react with the NMDA receptor.

Current evidence suggests that antibodies arise in the CSF of patients with SLE through penetration of the blood brain barrier (BBB); the antibodies in the CSF are polyclonal and there is albumin in the CSF as well. To mimic this clinical scenario, we administered LPS to mice immunized with the multimeric peptide. LPS causes a BBB breach in the hippocampus. Once the antibody penetrates the brain parenchyma, pathology proceeds as a 2-step process. First, the antibody functions as an allosteric modulator of NMDA receptor signaling to cause excitotoxic death of some neurons. This occurs over the course of a week ${ }^{23}$. High-mobility group box chromosomal protein 1 (HMGB1) is secreted by activated or damaged neurons. Recent studies have demonstrated that HMGB1 binds to the NMDA receptor on surviving neurons where
C1q is targeted to the synapses and binds to HMGB1. HMGB1 therefore serves as the bridge between the damaged neurons and C1q, which is detected by microglia and targets the synapses for pruning ${ }^{24}$.

Binding of DNRAb to NMDA receptors leads to increased free calcium in the cell where it is taken up by mitochondria to buffer. A high level of calcium in the mitochondria promotes cellular respiratory system, thus ROS production. Because of the increased calcium concentration, the mitochondrial membrane potential collapses and the mitochondrial permeability transition pores open, resulting in the release of proapoptotic molecules like Cytc and apoptosis-inducing factor, leading to neuronal death. Concomitantly, calcium activates cytosolic enzymes including phospholipases, proteases, and endonucleases, which promote necrosis ${ }^{25}$.

In a recent study, DWEYS immunized mice with a forebrain deletion of GluN2B subunits displayed acute neural loss in hippocampal CA1, while GluN2A knockout mice were protected from the DWEYS neuropsychological phenotypes, suggesting the essential mediatory role of GluN2A subunit in SLE cognitive dysfunctions ${ }^{26}$.

The second phase of pathology starts at 4 weeks after LPS administration, at a time when antibody is no longer detectable in the brain, and is characterized by microglial activation and dendritic pruning of the surviving neurons. This pathology persists for as long as we have observed the mice and is dependent on the presence of microglia and C1q. It manifests with impaired spatial memory. To understand the basis for this impairment, we performed electrophysiologic studies. The pyramidal neurons in CA1 region of the hippocampus represent place cell activity. A neuron fires intensely within a given area and becomes silent in other parts. The area of neuronal firing is known as a place field. Place fields from spatial maps rely on NMDA receptors ${ }^{27}$. The place fields are expanded in Balb/C mice with DNRAb penetration into the hippocampus, indicating a disrupted CA1 place cell system as a key neural substrate for DNRAb-mediated pathology ${ }^{28}$.

We also performed $18 \mathrm{~F}$-fluorodeoxyglucose micro positron emission tomography (FDG-microPET) in these mice to plot changes in brain metabolism. An inverse relationship was observed between neuronal number and regional metabolism compared to the positive correlation seen in control mice.

Because we found that depletion of microglia can prevent the loss of dendritic arborization or can reverse established injury, we asked whether suppressing microglial activation might also be effective. Treatment of mice with an angiotensin-converting enzyme inhibitor (ACEi) that penetrates the BBB and suppresses microglial activation spares cognitive function when given early or late after LPS administration ${ }^{29}$. This observation is of great importance as it suggests that there is sufficient neuronal plasticity to recover from the DNRAb-mediated insult.

Personal non-commercial use only. The Journal of Rheumatology Copyright $\subset$ $\odot 2020$. All rights reserved. 


\section{Neuroimaging}

Neuroimaging techniques constitute a promising method to objectively assess cognitive dysfunction in SLE and a potentially useful metric in clinical trials.

Several anatomical and functional neuroimaging modalities have been used in NPSLE, including diffusion tensor imaging (DTI), functional magnetic resonance imaging (fMRI), and PET scans ${ }^{30}$.

DTI is a noninvasive, refined MRI technique that detects the diffusion of water in brain tissue, allowing study of the brain structure and assessment of white matter (WM) integrity measured by fractional anisotropy $(\mathrm{FA})^{31}$. Several studies showed various WM correlates of NPSLE in SLE patients with and without acute NPSLE manifestations ${ }^{32,33}$.

The advantage of fMRI is its ability to detect variations in the deoxyhemoglobin levels in neurons as a measure of neuronal activity, serving as an indirect assessment of cerebral functions such as working memory, executive function, and attention ${ }^{34}$. Different studies demonstrated abnormalities in the hippocampal/parahippocampal regions of patients with SLE both at rest and during a memory task ${ }^{35,36}$.

The FDG-PET measures the uptake of glucose by the brain, serving as a measure of brain metabolic activity. The glucose metabolism in the brain can be affected by any inflammatory state as well as changes in neuronal density and activity. Therefore, FDG-PET provides a highly sensitive assessment tool for brain pathology $y^{37,38}$.

\section{Bridging Mouse Models to Human Disease}

To understand whether the mouse model can inform us about patients, we performed FDG-PET scans of patients with SLE who had no prior evidence of neuropsychiatric disease. We observed a higher resting metabolism in several areas of the brain ${ }^{39}$. These areas of hypermetabolism correlated with the serum DNRAb titers and memory impairment.

To validate these findings, we also assessed FDG-PET images in a larger cohort of patients with SLE. A total of 20 patients with SLE underwent FDG-PET and DTI imaging at baseline and at 15 months $^{40}$. This study was able to reproduce the initial finding of resting hypermetabolism in the hippocampus, orbitofrontal cortex, and basal ganglia as well as identify 3 new regions: sensorimotor cortex, and occipital and temporal lobes. Hypermetabolism in 5 of these regions correlated with poor performance on a memory test. Further, we showed a significant correlation between serum DNRAb, the performance on a spatial navigation task, and resting glucose metabolism in the anterior putamen and frontal cortex ${ }^{41}$. DTI images revealed the presence of regions of decreased microstructural integrity (measured by FA) structurally linked to the hypermetabolic regions. Tractography revealed that connecting tracts in the region of the hippocampus are substantially reduced in patients with SLE compared to healthy controls.

The structural and functional changes remained stable during the followup time of 15 months, suggesting that they possibly represent an "SLE-specific" pathology irrespective of disease activity and other confounders such as medications or prior central nervous system events. Taken together, the hippocampal hypermetabolism, decreased structural integrity of para-hippocampal regions, higher serum DNRAb, and poor performance on spatial memory testing suggest the possibility that the primary event takes place in the grey matter, leading to damage to WM tracts and spatial memory loss.

An $18-\mathrm{kDa}$ translocator protein referred to as TSPO is upregulated during microglial activation and therefore serves as a correlate of brain injury and inflammation. Consequently, it has been gaining interest as a target in neuroimaging of various neurodegenerative diseases ${ }^{42}$. Several second-generation radioligands, such as PBR28, have been developed for use in PET imaging providing a higher affinity for the TSPO. One study demonstrated a significant decrease in TSPO distribution in the hippocampus of patients with SLE compared to healthy controls and that these changes were more pronounced in SLE patients with $\mathrm{CI}^{43}$.

\section{Therapeutic Interventions}

The treatment of CI in SLE is exceedingly challenging, as is the case with NPSLE in general. The clinician is faced with several challenges starting with the attribution of CI to SLE-specific immune mechanisms as opposed to confounders or mimickers (medication side effects, infections, etc.) and objectively assessing the level of impairment. At this time, there is no clinically proven treatment that effectively targets CI in SLE.

As described above, there is significant evidence suggesting that microglia play a central role in the inflammatory cascade leading to CI in SLE. Therefore, a strategy that targets microglial activation, without inducing immunosuppression, is very attractive. The renin angiotensin system is a key player in neuroinflammation and is implicated in microglial activation ${ }^{44,45}$. Targeting this pathway using ACEi successfully inhibited microglial activation and neuronal damage in various neurodegenerative diseases ${ }^{46,47}$. A small study in older patients with Alzheimer disease (AD) showed that those patients receiving ACEi experienced a slower rate of cognitive decline. Several observational studies showed similar benefit of ACEi in patients with $\mathrm{AD}^{48,49}$.

Based on the promising results from the DNRAb-mediated mouse model of $\mathrm{CI}$ and in keeping with the commitment to find non-immunosuppressive therapy, we have designed a trial of ACEi in SLE patients with CI. The study is a phase II double-blinded randomized multicentral trial comparing the efficacy of lisinopril, an ACEi known to cross the BBB (or centrally acting) to benazepril, an ACEi that does not cross the BBB (or non-centrally acting). A major benefit of the trial is the use of objective assessment tools as endpoints. Patients will undergo FDG-PET imaging to

Personal non-commercial use only. The Journal of Rheumatology Copyright $(\subset) 2020$. All rights reserved. 
evaluate resting metabolism at baseline and at 12 months. Secondary endpoints will include changes on a battery of cognitive testing (spatial memory, spatial navigation, automated neuropsychological assessment metrics), patientreported outcomes, and disease activity measures. Further, the use of PBR28 tracer will allow for the study of microglial activation.

This trial would be the first study in humans, to our knowledge, to investigate the effectiveness of ACEi, a commonly used antihypertensive, in improving CI in patients with SLE, relying on advanced imaging techniques and objective assessment tools.

\section{Future Perspective}

Activation of microglia is implicated in the pathogenesis of CI in SLE, an NPSLE manifestation characterized by longterm progressive nature of neural degeneration. Detecting and validation of biomarkers based on neuroimaging studies is a fundamental step in the future clinical trials toward treatment of cognitive dysfunction in SLE.

Further research dealing with human and murine models of CI will promote a better understanding of the pathophysiological events that trigger and sustain this strong form of SLE disease and allow the possibility of finding therapeutic targets and an evidence-based approach for further treatment of the disease. We believe ACE inhibition is a rational potential therapeutic target in clinical trials to benefit CI.

Despite its major effect on the QOL of patients with SLE, CI in SLE remains widely understudied and poorly understood, and no targeted treatments are available. One of the major challenges of studying CI in SLE is the lack of objective assessment measures. We described several neuroimaging techniques that can be effectively used as metrics in clinical trials. We presented our experience with previous and ongoing clinical trials relying on sophisticated PET and MRI techniques to assess CI in SLE.

The study of the DNRAb-mediated mouse model of CI allowed us to identify the central role that microglial activation plays in this condition as well as to test promising therapeutic targets. The use of centrally acting ACEi appears to be an encouraging approach because it is mechanistically plausible, the drug has a well-established safety profile, and most of all, it is non-immunosuppressive.

\section{REFERENCES}

1. The American College of Rheumatology nomenclature and case definitions for neuropsychiatric lupus syndromes. Arthritis Rheum 1999;42:599-608.

2. Unterman A, Nolte JES, Boaz M, Abady M, Shoenfeld Y, Zandman-Goddard G. Neuropsychiatric syndromes in systemic lupus erythematosus: a meta-analysis. Semin Arthritis Rheum 2011;41:1-11.

3. Hanly JG, Urowitz MB, Su L, Bae SC, Gordon C, Wallace DJ, et al. Prospective analysis of neuropsychiatric events in an international disease inception cohort of patients with systemic lupus erythematosus. Ann Rheum Dis 2010;69:529-35.
4. Appenzeller S, Cendes F, Costallat LT. Cognitive impairment and employment status in systemic lupus erythematosus: a prospective longitudinal study. Arthritis Rheum 2009;61:680-7.

5. Calderón J, Flores P, Aguirre JM, Valdivia G, Padilla O, Barra I, et al. Impact of cognitive impairment, depression, disease activity, and disease damage on quality of life in women with systemic lupus erythematosus. Scand J Rheumatol 2017;46:273-80.

6. Lupus: Patient Voices. Report on Externally-led Patient-Focused Drug Development Meeting: September 25, 2017. [Internet. Accessed May 13, 2020.] Available from: https://s3 .amazonaws. com/stg.files.lupus.org/public/images/Advocacy/Documents/ Lupus\%20-\%20Patient\%20Voices\%20Report.pdf

7. Andrews BS, Eisenberg RA, Theofilopoulos AN, Izui S, Wilson $\mathrm{CB}, \mathrm{McC}$ onahey PJ, et al. Spontaneous murine lupus-like syndromes. Clinical and immunopathological manifestations in several strains. J Exp Med 1978;148:1198-215.

8. Jeltsch-David H, Muller S. Neuropsychiatric systemic lupus erythematosus and cognitive dysfunction: the MRL-lpr mouse strain as a model. Autoimmun Rev 2014;13:963-73.

9. Dixon FJ, Andrews BS, Eisenberg RA, McConahey PJ, Theofilopoulos AN, Wilson CB. Etiology and pathogenesis of a spontaneous lupus-like syndrome in mice. Arthritis Rheum 1978;21 Suppl 5:S64-7.

10. Santiago-Raber ML, Kikuchi S, Borel P, Uematsu S, Kaira S, Kotzin BL, et al. Evidence for genes in addition to Tlr7 in the Yaa translocation linked with acceleration of systemic lupus erythematosus. J Immunol 2008;181:1556-62.

11. Murphy ED, Roths JB. A Y chromosome associated factor in strain BXSB producing accelerated autoimmunity and lymphoproliferation. Arthritis Rheum 1979;22:1188-94.

12. Deane JA, Pisitkun P, Barrett RS, Feigenbaum L, Town T, Ward JM, et al. Control of toll-like receptor 7 expression is essential to restrict autoimmunity and dendritic cell proliferation. Immunity 2007;27:801-10.

13. Zhou T, Edwards CK 3rd, Yang P, Wang Z, Bluethmann H, Mountz JD. Greatly accelerated lymphadenopathy and autoimmune disease in 1 pr mice lacking tumor necrosis factor receptor I. J Immunol 1996;156:2661-5.

14. Zhou T, Bluethmann H, Eldridge J, Berry K, Mountz JD. Origin of CD4-CD8-B220+ T cells in MRL-lpr/lpr mice. Clues from a T cell receptor beta transgenic mouse. J Immunol 1993;8 Pt 1:3651-67.

15. Gilkeson GS, Ruiz P, Pritchard AJ, Pisetsky DS. Genetic control of inflammatory arthritis and glomerulonephritis in congenic lpr mice and their F1 hybrids. J Autoimmun 1991;4:595-606.

16. Gilkeson GS, Ruiz P, Grudier JP, Kurlander RJ, Pisetsky DS. Genetic control of inflammatory arthritis in congenic lpr mice. Clin Immunol Immunopathol 1989;53:460-74.

17. Steinberg AD, Roths JB, Murphy ED, Steinberg RT, Raveche ES. Effects of thymectomy or androgen administration upon the autoimmune disease of MRL/Mp-lpr/lpr mice. J Immunol 1980;125:871-3.

18. Izui S, Kelley VE, Masuda K, Yoshida H, Roths JB, Murphy ED. Induction of various autoantibodies by mutant gene lpr in several strains of mice. J Immunol 1984;133:227-33.

19. Luciano-Jaramillo J, Sandoval-García F, Vázquez-Del Mercado M, Gutierrez-Mercado YK, Navarrio-Hernandez RE, Martinez-Garcia EA, et al. Downregulation of hippocampal NR2A/2B subunits related to cognitive impairment in a pristane-induced lupus BALB/c mice. PLoS One 2019;14:e0217190.

20. Nakamura K, Xiu Y, Ohtsuji M, Sugita G, Abe M, Ohtsuji N, et al Genetic dissection of anxiety in autoimmune disease. Hum Mol Genet 2003;12:1079-86.

21. Shiozawa S, Kuroki Y, Kim M, Hirohata S, Ogino T. Interferon-alpha in lupus psychosis. Arthritis Rheum 1992; 35:417-22.

Personal non-commercial use only. The Journal of Rheumatology Copyright @ 2020 . All rights reserved. 
22. Gaynor B, Putterman C, Valadon P, Spatz L, Scharff MD, Diamond B. Peptide inhibition of glomerular deposition of an anti-DNA antibody. Proc Natl Acad Sci U S A 1997;94:1955-60.

23. Kowal C, DeGiorgio LA, Nakaoka T, Hetherington H, Huerta PT, Diamond B, et al. Cognition and immunity; antibody impairs memory. Immunity 2004;21:179-88.

24. Arinuma Y, Volpe BT, Nestor J, Fujieda Y, Kowal C, Diamond B. HMGB1 mediating C1Q adhesion on dendritic synapses of cultured cortical neurons through activated NMDA receptor. [Internet. Accessed April 23, 2020.] Available from: www.jns-journal.com/ article/S0022-510X(17)31298-4/fulltext

25. Bendorius M, Po C, Muller S, Jeltsch-David H. From systemic inflammation to neuroinflammation: the case of neurolupus. Int $\mathbf{J}$ Mol Sci 2018;19:3588.

26. Chan K, Nestor J, Huerta TS, Certain N, Moody G, Kowal C, et al. Lupus autoantibodies act as positive allosteric modulators at GluN2A-containing NMDA receptors and impair spatial memory. Nat Commun 2020;11:1403.

27. Ekstrom AD, Meltzer J, McNaughton BL, Barnes CA. NMDA receptor antagonism blocks experience-dependent expansion of hippocampal "place fields". Neuron 2001;31:631-8.

28. Chang EH, Volpe BT, Mackay M, Aranow C, Watson P, Kowal $\mathrm{C}$, et al. Selective impairment of spatial cognition caused by autoantibodies to the n-methyl-d-aspartate receptor. EBioMedicine 2015;2:755-64.

29. Nestor J, Arinuma Y, Huerta TS, Kowal C, Nasiri E, Kello N, et al. Lupus antibodies induce behavioral changes mediated by microglia and blocked by ACE inhibitors. J Exp Med 2018;215:2554-66.

30. Jeltsch-David H, Muller S. Neuropsychiatric systemic lupus erythematosus: pathogenesis and biomarkers. Nat Rev Neurol 2014;10:579-96.

31. Emmer BJ, Veer IM, Steup-Beekman GM, Huizinga TWJ, van der Grond J, van Buchem MA. Tract-based spatial statistics on diffusion tensor imaging in systemic lupus erythematosus reveals localized involvement of white matter tracts. Arthritis Rheum 2010 62:3716-21.

32. Jung RE, Caprihan A, Chavez RS, Flores RA, Sharrar J, Qualls CR, et al. Diffusion tensor imaging in neuropsychiatric systemic lupus erythematosus. BMC Neurol 2010;10:65.

33. Jung RE, Chavez RS, Flores RA, Qualls C, Sibbitt WL Jr., Roldan CA. White matter correlates of neuropsychological dysfunction in systemic lupus erythematosus. PLoS One 2012;7:e28373.

34. Mak A, Ren T, Fu EH, Cheak AA, Ho RC. A prospective functional MRI study for executive function in patients with systemic lupus erythematosus without neuropsychiatric symptoms. Seminars in arthritis and rheumatism. 2012;41:849-58.

35. Zhu C-M, Ma Y, Xie L, Huang JZ, Sun ZB, Duan SX, et al. Spatial working memory impairment in patients with non-neuropsychiatric systemic lupus erythematosus: a blood-oxygen-level dependent functional magnetic resonance imaging study. J Rheumatol 2017;44:201-8.
36. Liu S, Cheng Y, Xie Z, Lai A, Lv Z, Zhao Y, et al. A conscious resting state fMRI study in SLE patients without major neuropsychiatric manifestations. Front Psychiatry 2018;9:677.

37. Lee SW, Park MC, Lee SK, Park YB. The efficacy of brain (18)F-fluorodeoxyglucose positron emission tomography in neuropsychiatric lupus patients with normal brain magnetic resonance imaging findings. Lupus 2012;21:1531-7.

38. Weiner SM, Otte A, Schumacher M, Klein R, Gutfleisch J, Brink $\mathrm{I}$, et al. Diagnosis and monitoring of central nervous system involvement in systemic lupus erythematosus: value of F-18 fluorodeoxyglucose PET. Ann Rheum Dis 2000;59:377-85.

39. Mackay M, Tang CC, Volpe BT, Aranow C, Mattis PJ, Kroff RA, et al. Brain metabolism and autoantibody titres predict functional impairment in systemic lupus erythematosus. Lupus Sci Med 2015;2:e000074.

40. Mackay M, Vo A, Tang CC, Small M, Anderson E, Ploran EJ, et al. Metabolic and microstructural alterations in the SLE brain correlate with cognitive impairment. JCI Insight 2019;4:e124002.

41. Ploran E, Tang C, Mackay M, Small M, Anderson A, Storbeck J, et al. Assessing cognitive impairment in SLE: examining relationships between resting glucose metabolism and anti-NMDAR antibodies with navigational performance. Lupus Sci Med 2019;6:e000327.

42. Turkheimer FE, Rizzo G, Bloomfield PS, Howes O, Zanotti-Fregonara P, Bertoldo A, et al. The methodology of TSPO imaging with positron emission tomography. Biochem Soc Trans 2015;43:586-92.

43. Wang Y, Coughlin JM, Ma S, Endres CJ, Kassiou M, Sawa A, et al. Neuroimaging of translocator protein in patients with systemic lupus erythematosus: a pilot study using [(11)C]DPA-713 positron emission tomography. Lupus 2017;26:170-8.

44. Wright JW, Harding JW. The brain renin-angiotensin system: a diversity of functions and implications for CNS diseases. Pflugers Arch 2013;465:133-51.

45. Moreau ME, Garbacki N, Molinaro G, Brown NJ, Marceau F, Adam A. The kallikrein-kinin system: current and future pharmacological targets. J Pharmacol Sci 2005;99:6-38

46. Gebre AK, Altaye BM, Atey TM, Tuem KB, Berhe DF. Targeting renin-angiotensin system against Alzheimer's disease. Front Pharmacol 2018;9:440.

47. Dong YF, Kataoka K, Tokutomi Y, Nako H, Nakamura T, Toyama $\mathrm{K}$, et al. Perindopril, a centrally active angiotensin-converting enzyme inhibitor, prevents cognitive impairment in mouse models of Alzheimer's disease. FASEB J 2011;25:2911-20.

48. Hajjar IM, Keown M, Lewis P, Almor A. Angiotensin converting enzyme inhibitors and cognitive and functional decline in patients with Alzheimer's disease: an observational study. Am J Alzheimers Dis Other Demen 2008;23:77-83.

49. O’Caoimh R, Healy L, Gao Y, Svendrovski A, Kerins DM, Eustace $\mathrm{J}$, et al. Effects of centrally acting angiotensin converting enzyme inhibitors on functional decline in patients with Alzheimer's disease. J Alzheimers Dis 2014;40:595-603. 